\title{
Technological Paradigms and Firms' Interaction
}

\author{
Rainer Andergassen ${ }^{1}$, Franco Nardini ${ }^{2}$, Massimo Ricottilli $^{3}$ \\ ${ }^{1}$ Department of Economics, University of Bologna \\ Piazza Scaravilli 2, 40126, Bologna, Italy \\ e-mail: anderga@economia.unibo.it \\ ${ }^{2}$ Department of Mathematics for Social Sciences \\ Viale Filopanti 5, 40126 Bologna, Italy \\ e-mail: nardini@dm.unibo.it \\ ${ }^{3}$ Department of Economics, University of Bologna \\ Piazza Scaravilli 2, 40126, Bologna, Italy \\ e-mail: ricottilli@economia.unibo.it
}

June 4, 2003

\begin{abstract}
This paper deals with the aggregate effects of small, exogenous but idiosyncratic technological shocks on locally interacting firms. Its main purpose is to model a situation in which technological paradigms emerge through endogenous propagation and diffusion of information leading to an aggregate pattern. We develop a theoretical framework in which large technological correlations emerge due to localised interaction of single firms.

The paper states some simple results on spill-over dynamics determined by firms trying to improve their current technology and thus generating new information through investment in R\&D and through localised technological search. The first part shows that different growth regimes can arise from the general framework of interaction that we propose. The second part shows that an interesting regime characterised both by long run innovation growth and endogenous short run fluctuations emerges spontaneously.
\end{abstract}

Keywords: Local interaction, Spill-over Dynamics, Growth, Fluctuations

JEL: O33, O40 


\section{Introduction}

The source of volatility of aggregates is one of the most debated and open questions of economic analysis in many decades. The conventional response is that in order to generate a business cycle aggregate shocks are needed since independent and idiosyncratic ones cancel out in the aggregate due to the law of large numbers (see e. g. Long Plosser, 1983, Dupor, 1999). If this were the case, system wide phenomena such as innovation waves and technological convergence would never occur since technological innovation is the foremost example of an idiosyncratic occurrence typically affecting the economic system at the individual firm level.

Recent contributions have challenged the above mentioned conventional wisdom. Horvath $(1998,2000)$ has shown that sector specific shocks may cause aggregate fluctuations only when those involved are important input suppliers, thus recognising that firms do affect eachother at least through an economy's input-output structure. Contributions dealing with self organised criticality have provided a very interesting insight on the impact of exogenous events on systems populated by heterogeneous elements capable of interacting. Bak et al. (1993), Arenas et al. (2002), and Andergassen Nardini (2002) have proposed multi-firm, multi-sector models in which the effect of small independent shocks hitting single units do not cancel out in the aggregate owing to significant non-linear, strongly localised interactions between different members of the economy.

In this paper, we wish to inquire on the impact upon an economic system of idiosyncratic shocks which affect individual firms. The latter are heterogeneous, differently capable in respect to available technology, are rationally bounded, learn and need, therefore, to collect information to lay out their economic plans, in particular in order to technically improve. It is a consequence of these 'real world' assumptions that firms' knowledge base can possibly widely diverge determining the degree and strength of interaction and, thus, firm specific neighbourhoods of comprehension. It is within such neighbourhoods that information travels.

In Section 2 we show that, for critical values of the interaction strength between firms, even extremely small exogenous and idiosyncratic shocks of a technological nature may generate long run positive growth of the aggregate technological state of the economy yet exhibiting wide, short run oscillations. In Section 3 we identify, by taking into account their different levels of entropy, the neighbourhood which is capable to provide the strongest interaction and by a mean field approximation we show that information propagating through minimal entropy neighbourhoods may generate innovation waves initiated by the input of a single bit of information at the individual firm level.

A final section draws the conclusions. 


\section{Growth regimes: a general framework}

We consider an economy, populated by $n$ firms, $n$ very large. Each firm $i$ is characterised by a stochastic process $\phi_{i}$ which compounds endogenous and exogenous technological adjustments occurring as a direct consequence of gathering information on technological innovation. Endogenous adjustments are direct consequences of technological information spillovers generated inside the system, while exogenous adjustments are autonomously generated by the firm's R\&D activity ${ }^{1}$. Exogenous adjustments occur according to $\delta_{i}$, the latter being a random variable measuring the number of adjustments in the time unit which is assumed as i.i.d. with mean $\frac{\mu}{n}$ and variance $\sigma^{2}$. $\mu$ turns out to be the average aggregate number of exogenous adjustments generated by firms' R\&D activity in the unit time.

We assume that each firm i interacts with firms $j \in \aleph_{i}$, where $\aleph_{i}$ is the neighbourhood of firm $i$. Neighbourhoods are so defined as being strictly independent to generate random transmission of information: there is, therefore, no overlapping. This assumption can also be taken as a first order approximation, higher order correlations being neglected, of more complex interactive structures. In what follows we will take the neighbourhood structure as given, while in Section 3 we will show how these neighbourhoods can suitably be defined. Because of bounded rationality, we assume that firms observe only a limited number of firms, i.e. $\left|\aleph_{i}\right|<<n$. Within such a neighbourhood a firm $i$ receives information from firm $j$. We define $\varepsilon_{i, j}$ as a measure of interaction between neighbouring firms $i$ and $j$. As a consequence, $\varepsilon_{i, j} \delta_{j}$ is a random variable indicating the number of adjustments performed by firm $i$ in the time unit induced by adjustments of neighbouring firm $j$.

Given these assumptions, we obtain the following structure for the stochastic process leading to technology upgrading $d \phi_{i}$.

$$
d \phi_{i}=\delta_{i} d t+\sum_{j \in \aleph_{i}} \varepsilon_{i, j}\left(\delta_{j} d t+\sum_{k \in \aleph_{j}} \varepsilon_{j, k}\left(\delta_{k} d t+\sum_{l \in \aleph_{k}} \varepsilon_{k, l}\left(\delta_{l} d t+\sum \ldots\right)\right)\right)
$$

According to (1a) we have that each firm is able, in each point in time, to improve technologically either because of exogenous forces or because of endogenous spillovers from neighbouring firms. Notice that since $\mu>0$ there is a positive drift in the exogenous driving force. We measure each firm's state of technology by an appropriate productivity index $Y_{i}=e^{\pi \phi_{i}}$, where $\pi$ is the productivity rate of growth. Assuming additivity across firms, the aggregate technological state is represented by $Y=\sum_{i=1}^{n} e^{\pi \phi_{i}}$. We begin our enquiry by

\footnotetext{
${ }^{1}$ It is to be stressed that neither a single information spillover nor mere R\&D activity necessarily generate a technological upgrading, as will be shown in section 3 .
} 
studying the aggregate innovation growth rate $\gamma_{y}=\frac{d y}{d t}=\frac{d Y}{Y} \frac{1}{d t}$, given by

$$
\gamma_{y}=\pi \sum_{i=1}^{n} \eta_{i} \frac{d \phi_{i}}{d t}
$$

where $\eta_{i}=\frac{e^{\pi \phi_{i}}}{\sum_{i=1}^{n} e^{\pi \phi_{i}}}$. In order to study the short and long run behaviour of technology growth in this economy we substitute (1a) into (2) and calculate its average value and variance. For the following, however, we are going to assume that in the long run all firms share the same technology level, i.e. $e^{\phi_{i}}=e^{\phi_{j}}$ for each $i, j=1, \ldots, n$ and that $\pi$ is given and normalised to 1 . This assumption is justified since fluctuations of $\eta$ 's are of higher order than $\gamma_{y}$ given that the market mechanism weeds out firms which fall too much behind the top technological level.

We can state the first result:

Theorem 1 The long run technology growth rate and the variance are given by

$$
\begin{gathered}
E\left(\gamma_{y}\right)=\frac{\mu}{n}\left(1+\sum_{j \in \aleph_{i}} \varepsilon_{i, j}\left(1+\sum_{k \in \aleph_{j}} \varepsilon_{j, k}\left(1+\sum_{l \in \aleph_{k}} \varepsilon_{k, l}\left(1+\sum \ldots\right)\right)\right)\right) \\
\operatorname{Var}\left(\gamma_{y}\right)=\sigma^{2} \frac{1}{n}\left(1+\sum_{j \in \aleph_{i}} \varepsilon_{i, j}^{2}\left(1+\sum_{k \in \aleph_{j}} \varepsilon_{j, k}^{2}\left(1+\sum_{l \in \aleph_{k}} \varepsilon_{k, l}^{2}\left(1+\sum \ldots\right)\right)\right)\right)
\end{gathered}
$$

The results stated in Theorem 1 are quite general since they depend on the particular structure and strength of correlation between single firms. Let us first consider a particular, but important case.

Corollary 2 Suppose $\varepsilon_{i, j}=0$ for each $i, j=1, \ldots, n$, then we have that

$$
\begin{gathered}
E\left(\gamma_{y}\right)=\frac{\mu}{n} \underset{n \rightarrow \infty}{\longrightarrow} 0 \\
\operatorname{Var}\left(\gamma_{y}\right)=\frac{\sigma^{2}}{n} \underset{n \rightarrow \infty}{\longrightarrow} 0
\end{gathered}
$$

Corollary 2 is an example of well known central limit theorems. It states that, if there is no interaction between single firms, then, as their number diverges towards infinity, fluctuations average out. Further, the aggregate technology growth rate is vanishing small. If, however, the driving force of information is such that $\frac{\mu}{n}>0$, then the technological growth rate is entirely exogenous and in this case occurs without fluctuations. This is the standard neo-classical growth framework, where the state of technology grows at the exogenously given parameter $\frac{\mu}{n}$.

Contrary to the standard neo-classical case, we are interested in technological growth taking place through endogenous diffusion of available information such 
that long ranged and highly volatile innovation waves are emergent features of the economy. Thus, in the next section we will study the case of non-negligible interaction, informational spillovers occurring between firms. It is interesting to remark how the standard results mentioned above change and resulting patterns vary as the strength of interaction between single firms is allowed to increase. We accordingly assume that $\varepsilon_{i, j}>0$ for at least some of $i, j=1, \ldots, n$.

\subsection{Strength of spill-over effects and interaction: the spe- cial case of a symmetric economy.}

In this section we will solve and study the results stated in Theorem 1 in a special case and we will make extensive use, throughout these notes, of the following definition of a symmetric economy.

Definition 3 A symmetric economy $\left(\Omega, g,\left\{\varepsilon_{i, j}\right\}\right)$ is defined as follows:

1. $\Omega$ indicates the state space of the economy, i.e. $x_{i} \in \Omega$, for each $i=1, \ldots, n$, and where $x_{i}$ indicates the state variable of firm $i$;

2. each firm interacts with other $g$ firms, where $2 \leq g<n$, i.e. $\left|\aleph_{i}\right|=g$ for each $i=1, \ldots, n$;

3. the strength of interaction determining the information spillover within each neighbourhood is given by $\varepsilon_{i, j}$, for each $i, j$ where $j \in \aleph_{i}$.

In turn, a special case of a symmetric economy is $(\Omega, g, \varepsilon)$ with constant strength of interaction $\varepsilon_{i, j}=\varepsilon$, for each $i, j$ where $j \in \aleph_{i}$. Using Definition 3 we can now characterise the technological growth rate.

Corollary 4 Consider a symmetric economy $(\Omega, g, \varepsilon)$ as defined in Definition 3, where $\varepsilon>0$, then the results stated in Theorem 1 simplify to the following

$$
\begin{aligned}
E\left(\gamma_{y}\right) & =\frac{\mu}{n} \frac{1-(\varepsilon g)^{n+1}}{1-\varepsilon g} \\
\operatorname{Var}\left(\gamma_{y}\right) & =\frac{\sigma^{2}}{n} \frac{1-\left(\varepsilon^{2} g\right)^{n+1}}{1-\varepsilon^{2} g}
\end{aligned}
$$

From Corollary 4 we can see that the long run growth rate is larger the larger is the strength of the information spill-over. Further, it is no longer obvious that a law of large number applies such that no aggregate fluctuations occur. The next Proposition studies the different possibilities.

Proposition 5 Let $\alpha$ and $\beta$ be arbitrary finite constants. Consider a symmetric economy $(\Omega, g, \varepsilon)$ where $\varepsilon>0$. The following growth regimes can be defined 1. If $0<\varepsilon<\frac{1}{g}, \mu>0$ and both independent of $n$, then

$$
\begin{gathered}
E\left(\gamma_{y}\right) \underset{n \rightarrow \infty}{\longrightarrow} 0 \\
\operatorname{Var}\left(\gamma_{y}\right) \underset{n \rightarrow \infty}{\longrightarrow} 0
\end{gathered}
$$


2. If $\frac{1}{g} \leq \varepsilon<\frac{1}{\sqrt{g}}, \mu>0$ and both independent of $n$, then

$$
\begin{aligned}
& E\left(\gamma_{y}\right) \underset{n \rightarrow \infty}{\longrightarrow} \infty \\
& \operatorname{Var}\left(\gamma_{y}\right) \underset{n \rightarrow \infty}{\longrightarrow} 0
\end{aligned}
$$

3. If $\varepsilon=\sqrt{\frac{1}{g}-\frac{\alpha}{n g}}$ while $\mu>0$ and independent of $n$, then

$$
\begin{gathered}
E\left(\gamma_{y}\right) \underset{n \rightarrow \infty}{\longrightarrow} \infty \\
\operatorname{Var}\left(\gamma_{y}\right) \underset{n \rightarrow \infty}{\longrightarrow} \frac{\sigma^{2}}{\alpha} \frac{e^{\alpha}-1}{e^{\alpha}}
\end{gathered}
$$

4. If $\frac{1}{g} \leq \varepsilon<\frac{1}{\sqrt{g}}$, while $\mu=n \beta(g \varepsilon)^{-n}$ then

$$
\begin{gathered}
E\left(\gamma_{y}\right) \underset{n \rightarrow \infty}{\longrightarrow} \frac{g \varepsilon}{g \varepsilon-1} \beta \\
\operatorname{Var}\left(\gamma_{y}\right) \underset{n \rightarrow \infty}{\longrightarrow} 0
\end{gathered}
$$

5. If $\varepsilon=\sqrt{\frac{1}{g}-\frac{\alpha}{n g}}$ and $\mu=n \beta(g \varepsilon)^{-n}$ then

$$
\begin{gathered}
E\left(\gamma_{y}\right) \underset{n \rightarrow \infty}{\longrightarrow} \frac{g \varepsilon}{g \varepsilon-1} \beta \\
\operatorname{Var}\left(\gamma_{y}\right) \underset{n \rightarrow \infty}{\longrightarrow} \frac{\sigma^{2}}{\alpha} \frac{e^{\alpha}-1}{e^{\alpha}}
\end{gathered}
$$

6. If $\varepsilon>\frac{1}{\sqrt{g}}$ while $\mu=n \beta(g \varepsilon)^{-n}$ then

$$
\begin{aligned}
& E\left(\gamma_{y}\right) \underset{n \rightarrow \infty}{\longrightarrow} \frac{g \varepsilon}{g \varepsilon-1} \beta \\
& \operatorname{Var}\left(\gamma_{y}\right) \underset{n \rightarrow \infty}{\longrightarrow} \infty
\end{aligned}
$$

7. If $\varepsilon>\frac{1}{\sqrt{g}}$ while $\mu>0$ both independent of $n$, then

$$
\begin{gathered}
E\left(\gamma_{y}\right) \underset{n \rightarrow \infty}{\longrightarrow} \infty \\
\operatorname{Var}\left(\gamma_{y}\right) \underset{n \rightarrow \infty}{\longrightarrow} \infty
\end{gathered}
$$

Proof. Consider first case 5. We substitute $\varepsilon=\sqrt{\frac{1}{g}-\frac{\alpha}{n g}}$ into the expression for the long run growth rate stated in Corollary4 and obtain in this way

$$
E\left(\gamma_{y}\right)=\frac{\mu}{n} g^{\frac{n}{2}} \frac{1-\sqrt{g}\left(1-\frac{\alpha}{n}\right)^{\frac{n+1}{2}}}{1-\sqrt{g}\left(1-\frac{\alpha}{n}\right)^{\frac{1}{2}}}
$$

Since $\left(1-\frac{\alpha}{n}\right)^{\frac{n+1}{2}} \underset{n \rightarrow \infty}{\longrightarrow} e^{-\frac{\alpha}{2}}$ and setting $\mu=n \beta g^{-\frac{n}{2}}$ we obtain the result stated in the proposition. 
Consider now the variance of the growth rate. Substituting $\varepsilon=\sqrt{\frac{1}{g}-\frac{\alpha}{n g}}$ into the expression for the long tun growth rate stated in Corollary 4 and obtain in this way

$$
\operatorname{Var}\left(\gamma_{y}\right)=\frac{\sigma^{2}}{n} \frac{1-\left(1-\frac{\alpha}{n}\right)^{n+1}}{1-\left(1-\frac{\alpha}{n}\right)}
$$

Since $\left(1-\frac{\alpha}{n}\right)^{n+1} \underset{n \rightarrow \infty}{\longrightarrow} e^{-\alpha}$ we obtain the result stated in the proposition.

Part 1., 2. 3. and 4. 6. And 7.are consequences of (3) and (4).

As shown in Proposition 5, if the economy is driven only by small idiosyncratic information shocks, and if each firm interacts locally with other g neighbouring firms, where the strength of the spill-over effects is constant across the firms, then we can identify seven growth regimes:

- Negligibly small long run growth without short run fluctuations, case 1).

- Infinite long run growth rate but without short run fluctuations, case 2).

- Infinite long run growth rate with large short run fluctuations, case 3).

- Positive, finite long run growth but without short run fluctuations, case 4).

- Positive, finite long run growth rate with large short run fluctuations case 5).

- Positive, finite long run growth rate with infinite fluctuations, case 6).

- Infinite long run growth with infinite fluctuations, case 7).

In the first growth regime, as the economy becomes ever larger $(n \rightarrow \infty)$, the same aggregate and exogenous adjustments, $\mu$, become negligible for each firm: the probability of making them tends to be nil. Moreover, the strength of the interaction is very small, it is lower than the probability of each firm contacting one of its equally reachable neighbours, $\frac{1}{g}$. In these circumstances, information being endogenously passed on is very scant and become lost in the process, each firm retaining what meagre information it gets. For the same reason the variance is also zero. No technological paradigm can, therefore, emerge.

The second growth regime considers the case in which, although the average exogenous adjustments tend to become very small, the strength of firm to firm interaction becomes sufficiently large to set off a process of information transmission such that the exogenous adjustments, no matter how small, are ever amplified. For very large economies, the resulting long run growth rate becomes infinite. Nevertheless, interaction is not quite as strong as to generate positive fluctuations. It can easily be checked from $\operatorname{Var}\left(\gamma_{y}\right)$ as a function of $\varepsilon$ that $\varepsilon=\frac{1}{\sqrt{g}}$ is the threshold before which fluctuations are dampened to zero but positive past it. 
The third growth regime considers the case of $\varepsilon$ becoming larger with $n$. As the latter becomes ever larger $(n \rightarrow \infty)$, the said threshold is reached and the variance becomes positive. Hence, interaction is now as strong as to generate an infinite technological growth rate through strong endogenous diffusion but large aggregate fluctuations emerge.

The fourth growth regime deals with the case in which exogenous adjustments are a function of both the size of the economy and the strength of interaction, the former becoming smaller the larger are the latter. This case highlights the situation of an economy in which size and relation between firms render the original adjustments of less significance, all the more so the greater are these two characteristics, pre-eminence being acquired by endogenous transmission. Notice that the average long run growth rate is much larger than the exogenous one which is vanishing small as the size of the economy is allowed to become very large. Assuming $\varepsilon$ to be below the $\frac{1}{\sqrt{g}}$ threshold, fluctuations are zero but the long run rate of technological growth is finite and positive, the negligible size of the exogenous adjustments being exactly offset by the strength of the interaction. Technological paradigms, created through the endogenous diffusion of information and involving eventually the whole economy, possibly emerge.

Case five has a similar profile but the strength of the interaction is exactly the critical threshold above which fluctuations become positive while the long run growth rate is finite. This case marks a critical state since it separates non-exploding, absorbing dynamics from irregular ones with positive variance (regimes 2. and 3. respectively).

Growth regime six shows that as $\varepsilon$ goes past the critical threshold, the average exogenous adjustments at single firm level $\frac{\mu}{n}$ vanishing with size, fluctuations become infinitely large on account of very strong interaction while growth regime seven generates infinite growth if $\mu$ is positive and independent of size with equally infinite fluctuations.

There is no a-priori reason to hold that technological progress should necessarily occur in the shape of any of the cases discussed above. It is, indeed, possible to state that the actual regime depends crucially on how the parameters entering Corollary 4 are structurally tuned. The history of economic development in large systems after the industrial revolution may suggest that the most likely case is growth regime five, featuring positive finite technological growth together with finite fluctuations, and possibly, but less likely, growth regime six in which positive growth is coupled to very large fluctuations. Many developing economies, on the other hand, may well be categorised by growth regime one in which both average growth and fluctuations are nil.

\section{Emergent Technological Paradigms}

In this section we proceed to describe single firms and their interaction capability. We consider an economy, populated by $n$ firms, $n$ very large. Each firm can either receive an exogenous bit of information or an endogenous one. In the former case, new information is borne directly by the firm, through successful 
investment in $\mathrm{R} \& \mathrm{D}$, while in the latter case information is obtained by continuously observing a limited number of "neighbouring" firms. The first step is to describe in detail the neighbourhood structure.

\subsection{The interaction structure}

Since the capability of understanding and processing information coming from a different firm and a more advanced technological context depends on the common knowledge basis, the transmission of such information depends on the strength of this shared knowledge, i.e. the potential intensity of their interaction, and the probability of actually passing on relevant information. Let this strength be measured by $\varepsilon_{i, j} \in[0,1]$ for any two different firms.

The measures of cognitive distance, or proximity, thus defined and empirically observable through a statistical procedure, allow, in turn, a rigorous definition of the cognitive neighbourhoods through which innovative information can pass through. We are going to assume symmetry between firms $i, j$. There are, therefore, $\frac{n(n-1)}{2}$ couplings which compose the set:

Definition $6 E=\left\{\varepsilon_{i, j} \mid i, j=1, \ldots, n\right\}$, where $|E|=\frac{n(n-1)}{2}$.

In this case too we wish to deal with symmetric economies $\left(\Omega, g,\left\{\varepsilon_{i, j}\right\}\right)$ (Definition 3). The set of all possible neighbours out of the total number $n$ of firms in the whole economy can be defined as follows:

Definition 7 The set of all possible neighbourhoods of a firm $i$ is defined as

$$
\Gamma^{i}=\left\{\gamma_{i, j}\right\}_{j=1}^{\left|\Gamma^{i}\right|}
$$

where $\left|\gamma_{i, j}\right|=g$ for each $j=1,2, \ldots,\left|\Gamma^{i}\right|$, and where $\left|\Gamma^{i}\right|=\frac{(n-1) !}{g !(n-g-1) !}$, this for each $i=1,2, \ldots, n$. The collection of all sets of possible neighbourhoods for each firm defines the space of neighbourhoods

$$
\Gamma=\left\{\Gamma^{i} \mid i=1,2, \ldots, n\right\}
$$

These definitions provide a map of cognitive neighbourhoods for each firm and for the entire set of firms. The set of neighbours in each $\Gamma^{i}$ are of varying informative capability for the firm on account of the cognitive heterogeneity of its members. It follows that a ranking of these neighbourhoods can be compiled on the grounds of how enabling they are from the point of view of their informative content, given the combination of probabilities $\varepsilon_{i, j}$. A convenient measure of such informative content and of the ease with which information percolates through to let the firm learn and cumulate knowledge for innovation is Shannon's entropy measure (Klir and Folger, 1988). This entropy measure is:

$$
M\left(\gamma_{i, j}\right)=-\sum_{k \in \gamma_{i, j}} \varepsilon_{i, k} \log _{2}\left(\varepsilon_{i, k}\right)
$$


Given all $M\left(\gamma_{i, j}\right) \in[0, \infty]$ for all $\gamma_{i, j} \in \Gamma^{i}$, it is possible to compute the minimum, thus identifying the firm's neighbourhood which is most capable of carrying information and which best enables it to learn.

Definition $8 \aleph_{i}$ is the neighbourhood which is most likely to provide significant innovative information and which is, therefore, the cognitively relevant neighbourhood for the diffusion of innovative technologies:

$$
\aleph_{i}=\arg \min _{\gamma_{i, j} \in \Gamma^{i}} M\left(\gamma_{i, j}\right)
$$

This definition allows us to identify a firm's relevant neighbours. The probabilities $\varepsilon_{i, j}$, measuring cognitive relationships, depend in each neighbourhood on the number of firms which are nested therein. This proposition follows directly from the very definition of neighbourhood as the locus of dense inter-firm externalities. Economic history and studies in the Marshallian tradition have provided plenty of evidence for this fact. In particular, literature on industrial districts indicates that firms tend to cluster according to a predictable pattern often determined by agglomeration economies based on shared knowledge and know how. The greater is the number of firms in any given cluster, the greater is the cognitive correlation and the greater the probability that information spread across the cluster.

Conjecture 9 The probability that information be passed on is

$$
\varepsilon_{i, j}=\varepsilon=1-\frac{k}{n}
$$

\subsection{Innovation Dynamics}

We assume that the introduction of an innovation requires the accumulation of informational bits which is a process each firm has to complete if it wishes to do so. We consider a symmetric economy $(\Omega, g, \varepsilon)$, where $\varepsilon$ is the average strength of interaction ${ }^{2}$ and $g$ the number of informational bits each firm has to accumulate. In other words, we assume that more are the informational bits each firm has to gather, more are the neighbouring firms it continuously observes.

We characterise each firm according to the number of bits of information it has accumulated and we label the possible states (elements of the state space) as follows: $\Omega=\{0,1,2, \ldots, c, a\}$, where the cardinality of the state space $\Omega$ is $g+1$. Thus, $x_{i} \in \Omega$, for each $i=1, \ldots, n$, where $x_{i}$ indicates the state which characterises firm $i$. 0 indicates that the firm has just upgraded its technology, and it has no new information; 1 indicates that the firm has accumulated one bit of information and so on. Finally, $c$ indicates the state where it needs just one more bit of information such that technology upgrading becomes viable and $a$ indicates the active state where the firm upgrades its technology level.

\footnotetext{
tion.

${ }^{2}$ This is consistent with the mean field approach used to describe the diffusion of informa-
} 
We assume an average aggregate exogenous inflow of information $\mu$. We are interested in the case where technological paradigms are emergent features, and symmetric to the previous part of these notes, this will occur only if the time scale of the exogenous information inflow is very slow compared to diffusion dynamics, i.e. $\mu \rightarrow 0$.

The dynamics are as follows: given that a firm is in a state $o$, if it receives a bit of information (either an exogenous or an endogenous one) it switches to state $o+1$. If state $o+1<a$ nothing happens until the next bit of information arrives. On the other hand, if $o+1=a$, then it upgrades its technology, and transfers in this way a bit of information with probability $\varepsilon$ to $g$ neighbouring firms. Notice that since each firm always observes the same neighbourhood, nothing happens until a new innovation is introduced. Only once technology upgrading occurs, will information accumulated by the innovating firm be freed to some degree. Thus, as long as the accumulation of information continues, acquired knowledge remains tacit, and cannot help other firms introduce new innovations.

Vespignani and Zapperi (1998) show that the mean-field approximation to the interaction between single firms well approximates stationary state dynamics. Thus, we cluster firms according to their state. We call $\rho_{o}$ the average density of firms being in state $o$, where $o=1,2, \ldots, c, a$.

The state space dynamics are described by the following master equations

$$
\begin{gathered}
\dot{\rho}_{a}=-\rho_{a}+\rho_{c}\left(\mu+g \varepsilon \rho_{a}\right) \\
\dot{\rho}_{c}=-\rho_{c}\left(\mu+g \varepsilon \rho_{a}\right)+\rho_{c-1}\left(\mu+g \varepsilon \rho_{a}\right) \\
\ldots \\
\dot{\rho}_{1}=-\rho_{1}\left(\mu+g \varepsilon \rho_{a}\right)+\rho_{0}\left(\mu+g \varepsilon \rho_{a}\right)
\end{gathered}
$$

The first term of the first differential equation in (5) indicates the outflow of firms from the active technology upgrading state: once a firm introduces a new technology it switches immediately to state 0 and starts the information collection process from the beginning. The second expression indicates the inflow of firms into the active state: firms being in state $c$, receiving either an exogenous bit of information (with probability $\mu$ ) or an endogenous one (with probability $\left.g \varepsilon \rho_{a}\right)$, switch to the active state. The other expressions can be interpreted in a similar way.

Since $\rho_{o}$, for $o=1,2, \ldots, c, a$ are average densities, the following normalisation condition has to be satisfied:

$$
\rho_{0}+\rho_{1}+\ldots+\rho_{c}+\rho_{a}=1
$$

It can be shown that the stationary state is asymptotically stable (see Vespignani and Zapperi, 1998). We are interested in the stationary average number of firms introducing a new technology, given that a single, small idiosyncratic exogenous informational bit hits the economy. 
Proposition 10 If we call $x$ the number of firms introducing a new technology, then the stationary average number of firms innovating, given that a single, small idiosyncratic informational bit hits the economy, is given by

$$
E(x)=\frac{1}{g(1-\varepsilon)}
$$

Proof. Consider first the conditional stationary average number of firms introducing an innovation. Given that a single firm introduces a new innovation, the average number of firms innovating is given by

$$
E\left(x \mid x_{i}=a\right)=1+g \varepsilon \rho_{c}+\left(g \varepsilon \rho_{c}\right)^{2}+\left(g \varepsilon \rho_{c}\right)^{3}+\ldots
$$

In the limit where $n \rightarrow \infty$ this latter expression converges towards

$$
E\left(x \mid x_{i}=a\right)=\frac{1}{1-g \varepsilon \rho_{c}}
$$

It can be shown that, in the stationary state, $\lim _{\mu \rightarrow 0} \rho_{c}=\frac{1}{g}$, and as a consequence (6) becomes

$$
E\left(x \mid x_{i}=a\right)=\frac{1}{1-\varepsilon}
$$

Using the law of iterated expectation we obtain the result stated in the Proposition.

Notice that as long as $\varepsilon<1$, the average number of firms introducing an innovation remains finite, even though the number of firms diverges towards infinity. This implies that the average number of firms introducing an innovation is vanishing small compared to the total number of firms existing in the economy. In the latter case the correlation among the single firms remains small and as a consequence fluctuations average out in the process of aggregation.

On the other hand, if $\varepsilon \rightarrow 1$, then the number of firms introducing an innovation diverges towards infinity. In this case it is no longer obvious that the average number of firms introducing a new innovation is vanishingly small compared to the total number of firms existing in the economy.

If a new technology is introduced, the rate of increase of productivity is $\pi$. While taken as a constant independent of $g$ until now, is best understood as a function of the informative content implied by the innovation process. The greater is the latter, the higher is the likely productivity increase. Hence, we postulate that $\pi=\pi(g)$ and such that $\pi^{\prime}>0, \pi^{\prime \prime}<0$. Since the aggregate state of technology is given by $Y=\sum_{i=1}^{n} e^{\pi(g) \phi_{i}}$ and $d \phi_{i}=1\left(x_{i}=a\right) d t$, it follows from (2) and Proposition 10 given the assumptions made that the long run growth rate of technical progress is

$$
E\left(\gamma_{y}\right)=\frac{\pi(g)}{n} \frac{1}{g(1-\varepsilon)}
$$

We observe that if $\varepsilon<1$, then as the number of firms diverges towards infinity the long run growth rate becomes negligibly small. In this case the 
innovation avalanches are too small, and as a consequence their impact on the long run growth rate is negligible. Thus, no innovation waves and technological paradigms can emerge.

On the other hand if we make the Marshallian Conjecture 9 we obtain the following value for the long run growth rate

$$
E\left(\gamma_{y}\right)=\frac{\pi(g)}{g k}
$$

In this case the long run growth rate remains finite, even though the exogenous driving force is negligibly small. The spillover effects are such that innovation waves and technological paradigms are emergent features of the economy and growth in this case occurs through large fluctuations. Observe that the long run growth rate depends on structural parameters of the economy. In particular, the larger is the number of informational bits each firm has to accumulate such that the introduction of the innovation becomes viable, the lower is the long run growth rate of the economy.

\section{Conclusions}

This paper shows that structural characteristics matter. More specifically, growth and fluctuations depend on how each firm's neighbourhood is determined in terms of the number of firms which belong to it and of the interaction strength they are capable of. Transmission of exogenous, idiosyncratic shocks and the diffusion of innovation waves, avalanches, are a consequence of the crucial role they play. As the strength of interaction rises in respect to a neighbourhood firm population very different behaviour is obtained, in the limit of large systems, from negligible technological growth with no fluctuations to a very large size in both magnitudes through all possible intermediate cases.

Technological paradigms emerge in consequence of such interaction. The paper highlights the importance of neighbourhood transmission and of accumulation of information thereby made possible in determining the mean value and volatility of the avalanche size. It is finally interesting to note that at a microlevel neighbourhood size is the result of an evolutionary process; at a macro-level it concurs to determine the long run productivity growth rate. There is, however, a trade-off since while a large and information-wise rich neighbourhood increases technological growth, the effort of collecting such information weakens it. Whether there are positive or negative feedbacks such that the economy self organizes, or a suitable policy is necessary to drive the economy towards the optimal aggregate growth rate is an entirely open question. Further research will investigate this interesting point.

\section{References}

[1] Andergassen, R., Nardini, F.,(2002): 'Endogenous innovation waves and economic growth.' Working Paper n. 446, Department of Economics, University 
of Bologna. www.dse.unibo.it/wp/446.pdf.

[2] Arenas, A., Díaz-Guilera, A., Pérez, C. J., Vega-Redondo, F., (2002): 'Selforganized criticality in evolutionary systems with local interaction'. Journal of Economic Dynamics and Control 26, 2115-2142.

[3] Bak, P., Chen, K., Scheinkman, J., Woodford, M.,( 1993): 'Aggregate fluctuations from independent sectoral shocks: self organized criticality in a model of production and inventory dynamics.' Ricerche Economiche 47, 3-30.

[4] Dupor, B., (1999): 'Aggregation and irrelevance in multi sector models.' Journal of Monetary Economics 43, 391-409.

[5] Horvath, M. T. K., (1998): Cyclicality and sectoral linkages: aggregate fluctuations from sectoral shocks'. Review of Economic Dynanics 1, 781-808.

[6] Horvath, M. T. K.,(2000): 'Sectoral shock and aggregate fluctuations.' Journal of Monetary Economics 45, 69-106.

[7] Long, J., Plosser, C., (1983):' Real business cycles.' Journal of Political Economy 91, 39-69.

[8] Vespignani, A., Zapperi, S., (1998): 'How Self-organized criticality works: a unified mean field picture'. Physical Review E 57, 6345-6362. 